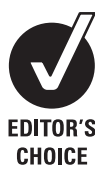

${ }^{1}$ Department of NeuroOphthalmology and Strabismology, Kantonsspital, St Gallen, Switzerland; ${ }^{2}$ University of Bern, Bern, Switzerland

Correspondence to: Professor D S Mojon, Department of NeuroOphthalmology and Strabismology, Kantonsspital, 9007 St Gallen, Switzerland; daniel.mojon@kssg.ch

Accepted 1 April 2009 Published Online First 2 June 2009

\title{
Saccade testing to distinguish between non-organic and organic visual-field restriction
}

\author{
M S Zinkernagel, ${ }^{1}$ N Pellanda, ${ }^{1}$ A Kunz, ${ }^{1}$ D S Mojon ${ }^{1,2}$
}

\begin{abstract}
Aim: The authors described and tested a simple bedside procedure to detect non-organic visual-field loss.

Methods: Prospective comparative observational case series of 16 patients with non-organic visual-field loss and 15 patients with organic visual-field loss were examined. Saccade patterns provoked by a stimulus outside the claimed visual field were assessed by a masked observer. Results: Whereas, in organic visual-field defects, eye movements as noted by the observer were in small and erratic searching patterns towards the visual-field defect in all patients (15/15), most patients with non-organic visual-field loss (14/16) were able to jump directly to the presented red cap in one directional large saccade, although the stimulus was outside their stated visual field. The sensitivity of the saccade test in detection of nonorganic visual-field loss by a masked observer was $87 \%$ (95\% Cl 60\% to $97 \%$ ) and the specificity was $100 \%$ (95\% $\mathrm{Cl} 75 \%$ to $100 \%)$. The positive predictive value for nonorganic visual-field loss of the saccade test was $100 \%$, and the negative predictive value was $90 \%$.
\end{abstract}

Conclusions: The saccade test is a quick and reproducible examination to use and is largely independent of the patient's willingness for cooperation. The authors believe that the test will be of value to clinicians on bedside evaluation when non-organic visual-field loss is suspected.

Non-organic visual-field loss is occasionally encountered by the ophthalmologist. It can be caused deliberately by feigning visual-field loss, socalled malingering, by a subconscious process, defined as ocular conversion disorder, ${ }^{1}$ lack of cooperation, somatisation disorders or psychiatric diagnoses.

Whatever the underlying cause, a high index of suspicion is needed to consider this condition, and it is often only after extensive neurological and ophthalmological workup and documentation that malingering or a psychogenic cause may be detected. $^{2}$ Thus, to prevent excessive medical investigations and shorten the period of work disability, it is useful to have tests which are easy to perform and reproducible, and have a high specifity to point towards this condition. Even if all the signs suggest a non-organic aetiology, it has to be kept in mind that in some cases non-organic disease may coexist with organic disease. ${ }^{3}$

Whereas a normal subject is able to calculate the distance to fixate an object recognised in the peripheral visual field and thus can trigger directional saccades, patients with hemianopsia or constricted visual fields are not able to localise the target stimulus in their retinal eccentricity and thus will display a searching eye movement pattern until they find the target. ${ }^{45}$ Most malingerers or patients with psychogenic visual-field loss, however, do not realise that testing the accuracy of their saccades corresponds to the function of their visual field and display a high accuracy in their saccadic eye movements, although the target is in a supposedly blind area.

Since saccadic eye movements can usually be observed with the naked eye, they allow insight into the patient's perceptual visual-field state. Although the test has been described in general ophthalmology textbooks on non-organic visual loss, ${ }^{6}$ there is, to our knowledge, no study validating its sensitivity and specificity. In order to address this issue, we sought to determine whether observation of saccade patterns allows differentiation between organic and non-organic visual-field defects by an masked observer and validation of the sensitivity and specificity of this test in a prospective study.

\section{MATERIAL AND METHODS}

The research followed the Tenets of the Declaration of Helsinki. It was considered to inform the patients about the study. However, this might have alerted the behaviour of the patients with non-organic visual-field loss. Therefore, and because of the completely noninvasive and short nature of the saccade testing, the ethical committee permitted the examination to be performed without the consent of the patient.

Inclusion criteria included consecutive patients seen by DSM in the years 2006 and 2007 in his private practice at the Kantonsspital St Gallen, having Goldmann kinetic perimetry for unclear visual-field defects. Since most of these patients were referrals from other ophthalmologists, the proportion of non-organic visual-field loss was not representative for typical ophthalmological demographics. Patients with organic visual-field loss were included if they had a structural abnormality in neuroimaging or eye examination explaining the pattern of visual-field loss.

Patients were defined as having non-organic visual-field loss if they had a normal structural eye exam, no abnormality on neuroimaging or electrophysiological testing to support an organic aetiology for the visual-field loss and/or evidence for non-organic visual loss through testing other than the saccadic testing. Standard full-field ERG and VEP were carried out in $69 \%(11 / 16)$ of cases with non-organic visual-field defects. MRI testing was performed in $62 \%(10 / 16)$ of patients. Some patients $(2 / 16,12.5 \%)$ did not have electrophysiological testing because Goldmann kinetic perimetry 
and history pointed unambiguously towards non-organic visualfield loss.

The "gold standard" for diagnosis of non-organic visual-field loss was assured by testing the visual field at various distances. Kinetic Goldmann perimetry (background luminance $10 \mathrm{~cd} / \mathrm{m}^{2}$ ) with various stimulus luminances according to the visual acuity was done in the usual fashion with the stimulus moved in from the periphery at the standard distance of $30 \mathrm{~cm}$. Then, the same procedure with the same settings was repeated at a double distance of $60 \mathrm{~cm}$. In patients with non-organic visual-field defects, the radius of the visual field was not significantly larger or even smaller when tested at double distance. In addition, spiral-shaped or tunnel-shaped visual fields in Goldmann perimetry were used as a criterion for diagnosis of non-organic visual-field loss.

In addition, spiral-shaped or tunnel-shaped visual fields in Goldmann perimetry were used as a criterion for diagnosis of non-organic visual-field loss.

Exclusion criteria for non-organic visual-field loss included an abnormal structural eye exam that could explain visual-field loss or abnormality on neuroimaging or electrophysiological testing in support of an organic aetiology for the visual-field loss or lack of a second test supporting non-organic visual-field loss. Exclusion criteria for patients with organic visual-field loss were: (1) inadequate clinical investigation and documentation, (2) insufficient evidence for organic visual loss or (3) patients who were not alert and oriented.

All saccade tests were performed by the same investigator (DSM), in order to reduce the testing procedure variability. He was aware of the final diagnosis, organic or non-organic visualfield loss, and had seen the results of the Goldmann visual-field testing or a global constriction of the visual field. In addition, a masked observer was present to evaluate the saccade pattern.

The examiner was sitting in front of the seated patient at a distance of about $60 \mathrm{~cm}$, holding in each hand a bottle of eyedrops with a red cap. All tests were performed binocularly, because all examined patients had binocular visual-field defects. The examiner's wrists were placed at the patient's eye level about $30^{\circ}$ to the right and left in a plane approximately $50 \mathrm{~cm}$ away from the patient (fig 1). The patient was informed about the test in the following way:

Now we will retest your eye movements, which were already previously found to be normal. I will show you a red bottle cap to your right. Please never move your head, just look at the target. Then, when I say "left," please look at the red bottle cap to your left. Continue to look at the left bottle cap until I say "right."

This procedure will be repeated several times.

While the patient was looking at one target, the position of the other bottle remained unchanged or not. If the position was changed, the wrist and arm were kept in the same position, and only the hand was flipped either up or down, depending on the actual bottle position (fig 2). This is very important, since any movement of the arm might be detected by an intact visual field and, thus, could represent an indirect indication as to where the target has been moved. Saccade testing was repeated several times, and the patient had at least $2 \mathrm{~s}$ to rest on one target before refixating the other target. To provide a masked observation, a third person, unaware of the patient's diagnosis, was observing the saccades between the four possible positions. A non-organic saccadic response in our study was defined as a single, accurate, and direct saccade to the presented target in the supposedly blind visual field as judged by an independent observer. An organic saccadic response was defined as an inaccurate or multistep searching movement towards a target in the blind field. The masked observer then judged whether all saccades were directional and accurate towards all stimuli demonstrating an intact visual field or if the saccades were in an erratic searching pattern, indicating a visual-field defect. If an abnormal visual field was suspected, the observer had to state which of the following field defects was suspected: quadrant (and if so which one), hemianopsia (and if so which part), only one quadrant intact (and if so which one), or a global constriction of the visual field. Only the result of the masked observer, but not the opinion of the person performing the saccade test (DSM), was used for analysis.

\section{RESULTS}

Thirty-one consecutive patients with visual-field restriction referred to the Neuro-Ophthalmological Service at the Kantonsspital St Gallen, Switzerland, between 2006 and 2007 were included. Visual-field defects were confirmed by standard Goldmann perimetry. Sixteen patients had non-organic visualfield loss (mean age: 41 years). Fifteen patients had organic visual-field loss (mean age: 40 years). All non-organic visualfield defects were concentric to $10^{\circ}$ of remaining visual field or less. Patients with organic visual-field loss had either quadrant or hemianopsia (12 patients), with absolute visual-field defects in that quadrant or hemifield, or concentric visual-field loss to $10^{\circ}$ of remaining visual field or less (three patients).

All patients had a thorough neuro-ophthalmological examination with funduscopy, biomicroscopy, ocular motility and pupil evaluation.

The mean visual acuity in the group with organic visual defects was $0.8 \mathrm{OD}$ and $0.85 \mathrm{OS}$ (range best-corrected visual acuity of the better eye (BCVA) in decimal fraction: 0.2-1.25), and in the group with non-organic visual-field loss, the mean visual acuity was $0.64 \mathrm{OD}$ and 0.62 OS (BCVA: 0.1-1.25).

All patients (15/15) with organic visual-field defects displayed at least two saccades towards the presented target and usually showed small and erratic searching patterns towards the visualfield defect. In contrast, saccadic eye movements towards the intact visual field were accurate. This searching pattern was found, whether patients had a truly constricted visual field or hemianopsia and regardless of the underlying organic condition,

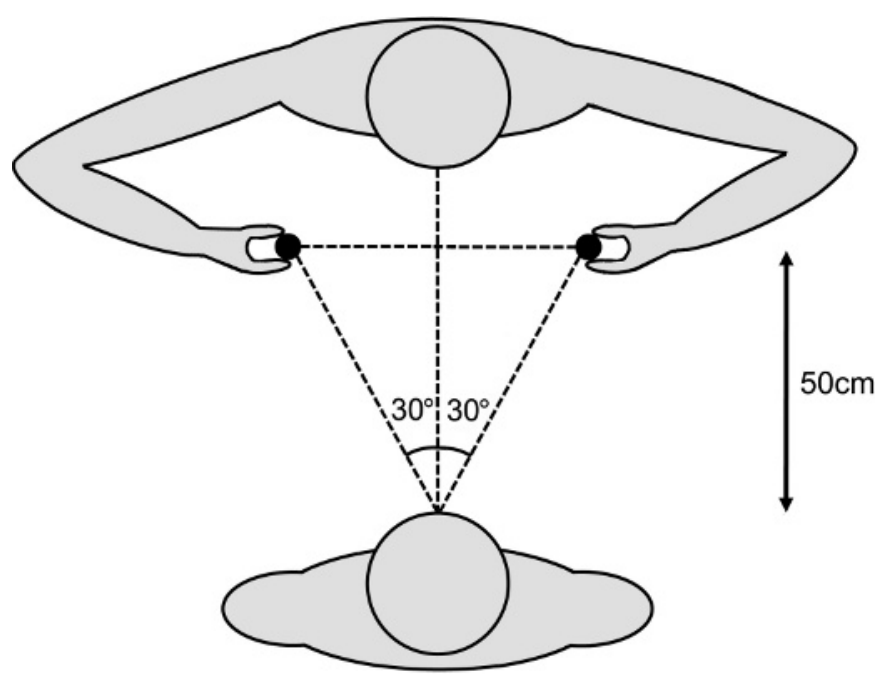

Figure 1 Test arrangement for saccade testing. The examiner's wrists were placed at the patient's eye level about $30^{\circ}$ to the right and left in a plane approximately $50 \mathrm{~cm}$ away from the patient. 


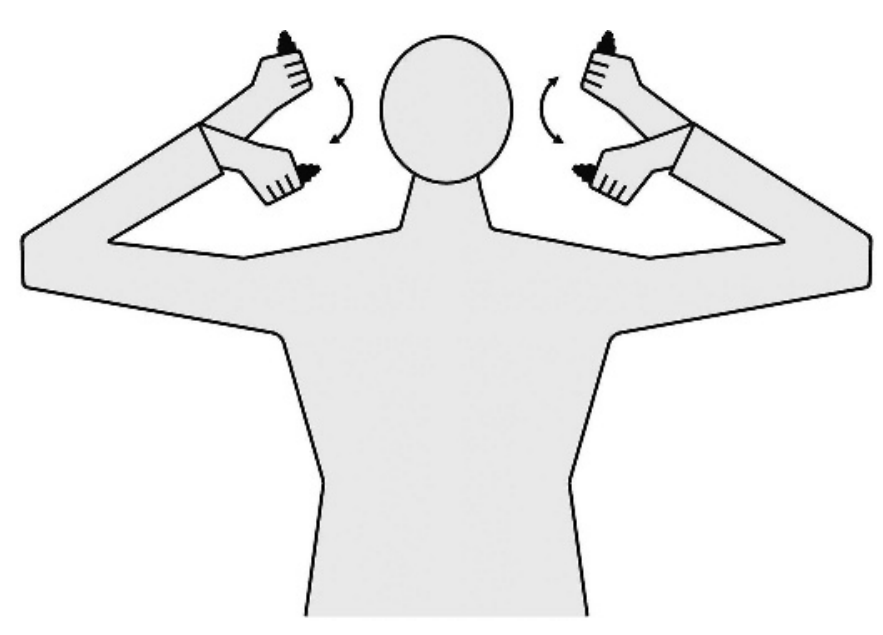

Figure 2 Test arrangement for saccade testing. While the patient was looking at one target, the position of the other target remained unchanged or not. If the position was changed, the wrist and arm were kept in the same position, and only the hand was flipped either up or down.

as long as the presented stimulus was outside their visual field. In contrast 14 out of 16 patients (87\%) with non-organic visualfield loss were able to jump directly to the presented finger in one directional large saccade, although the stimulus was outside their stated visual field. Two out of 16 patients (13\%) with nonorganic visual-field loss displayed saccadic patterns which were considered as erratic by the observer.

These two saccade patterns were highly reproducible in either group and appeared to be independent of visual acuity, as long as the presented stimulus could be recognised. The nature of the visual-field defect (i.e constrictive, quadrantic or hemifield) was identified correctly by the masked observer in all cases. It is important that the test be conducted using four different hand positions by the examiner (ie, left up and down and right up and down). Only with this method would the masked observer be able to differentiate between quadrantic or hemianopsia or constricted visual fields.

The sensitivity of the saccade test in detection of non-organic visual-field loss was $87 \%$ (95\%CI 60-97\%) and the specificity was $100 \%$ (95\%CI $75-100 \%$ ). The positive predictive value for non-organic visual-field loss of the saccade test was $100 \%$, and the negative predictive value was $90 \%$. Additionally three patients in this group displayed a spiral-shaped visual field highly suggestive of a non-organic constricted visual field.

\section{DISCUSSION}

Diagnosis of non-organic visual-field loss may be very difficult, especially when patients are malingering, have had multiple prior examinations or have rehearsed their visual-field defect. ${ }^{7}$ It has been shown that, with minimal coaching, healthy subjects can imitate quadrantic, hemianopic and altitudinal visual-field defects in both automated and manual perimetry. ${ }^{8}$ In most cases, however, non-organic visual-field defects are bilateral and concentric, because concentric defects appear to be easier to simulate. In addition, non-organic visual-field defects often present with other ocular manifestations of non-organic ocular disease. These include first and foremost loss of visual acuity ${ }^{9} 10$ but also paralysis of horizontal and vertical gaze, monocular diplopia, ptosis, blepharospasm, nystagmus, convergence and accommodation insufficiency, and spasms of the near reflex.
Whereas numerous special tests exist to assess non-organic loss of visual acuity ${ }^{11}$ there are few tests to assess non-organic visual-field restriction. A good test to convey non-organic visual field constriction is to repeat the Goldmann perimetry at double the original distance. Whereas most patients with an organically constricted visual field will display an increase in the absolute size of the visual field, patients with a non-organically constricted visual field will maintain about the same absolute size of their visual field. Monocular hemianopsia persisting on binocular testing will also arouse suspicion.

Although saccade testing is well known by many ophthalmologists and is described in general ophthalmology textbooks on non-organic visual loss, there is no report to validate its specificity and sensitivity.

We revisit this quick and reproducible test to use when nonorganic visual-field defects are suspected and which is largely independent of the patient's willingness for cooperation and determined its sensitivity and specificity in a prospective clinical study. The test makes use of the fact that erratic saccades are very difficult to simulate, and directional saccades are very hard to suppress. Thus, observation of triggered saccades allows a statement about the visual field beyond the claimed limits.

An important advantage of this test compared with others is that the patient is not suspicious that the visual field is tested. Often the patient will think that their eye movements and reaction time are examined. This feeling is reinforced by the examiner's statement that "the patient's eye movements, which were already previously found to be normal, will be retested."

This command will help eliminate false-negative answers and may explain the test's high negative predictive value of $90 \%$. Saccade testing can be repeated several times, and it is crucial not to browbeat the patient. In this context, it is also imperative that the patient have at least $2 \mathrm{~s}$ to rest on one target before refixating the other target. This allows the patient with a nonorganic visual-field loss to localise the target in the supposedly blind retinal eccentricity.

Especially when used with a masked third observer, the test may be a tool in fast and objective decision-making when a nonorganic aetiology of visual-field restriction is suspected.

Our test, however, also has several limitations and shortcomings. First, the test cannot be used in patients unable to hold fixation of the presented stimulus due to extremely decreased bilateral central visual acuity or nystagmus. In our study, all the patients were able to fixate and had sufficient visual acuity in at least one eye to detect the stimulus. Second, differentiating between absolute concentric non-organic visualfield loss and concentric visual-field loss with relative scotomas may present a problem. This has not been tested in this study. Patients with a relative concentric scotoma may be able to perform normal saccades towards the presented stimulus and thus may be mistaken as malingerers. In addition, the fact that the principal examiner was aware of the final diagnosis could have affected the course of the examination in unforeseen ways.

Although this method has a very high specifity in the diagnosis of non-organic visual-field defects, it should only be used as an extension of the complete neurological and neuroophthalmological examination to rule out an underlying organic component. Regarding the sensitivity of $87 \%$ of this test, the possibility that some patients with non-organic visual-field defects might be able to feign an erratic saccade pattern has to be kept in mind. Often referral to an ophthalmological clinic for specific testing depending on the nature of the visual-field loss and complaints of the patient might be necessary to confirm the diagnosis. 
Funding: The study was partly funded by the OPOS Foundation, St Gallen.

Competing interests: None.

Ethics approval: Ethics approval was provided by Ethikkommission Kantonsspital St Gallen.

Provenance and peer review: Not commissioned; externally peer reviewed.

\section{REFERENCES}

1. Miller BW. A review of practical tests for ocular malingering and hysteria. Surv Ophthalmol 1973;17:241-6.

2. Kathol RG, et al. Functional visual loss. Follow-up of 42 cases. Arch Ophthalmol 1983;101:729-35.

3. Keltner JL, et al. The California syndrome. Functional visual complaints with potential economic impact. Ophthalmology 1985:92:427-35.

4. Meienberg $\mathbf{0}$. Clinical examination of saccadic eye movements in hemianopia. Neurology 1983:33:1311-15.
5. Zangemeister WH, et al. Eye-head coordination in homonymous hemianopia. J Neurol 1982;226:243-54.

6. Weller M, Wiedemann P. Hysterical symptoms in ophthalmology. Doc Ophthalmol 1989:73:1-33.

7. Keane JR. Neuro-ophthalmic signs and symptoms of hysteria. Neurology 1982;32:757-62.

8. Thompson JC, Kosmorsky GS, Ellis BD. Field of dreamers and dreamed-up fields: functional and fake perimetry. Ophthalmology 1996;103:117-25.

9. Lim SA, Siatkowski RM, Farris BK. Functional visual loss in adults and children patient characteristics, management, and outcomes. Ophthalmology 2005;112:1821-8.

10. Bengtzen R, et al. The "sunglasses sign" predicts nonorganic visual loss in neuroophthalmologic practice. Neurology 2008;70:218-21.

11. Mojon DS, Flueckiger P. A new optotype chart for detection of nonorganic visual loss. Ophthalmology 2002:109:810-15.

\section{Education}

\section{ANSWERS}

From questions on page 1199

\section{What are the possible diagnoses based upon the patient's history?}

The history of painless transient monocular visual loss is consistent with an ischaemic event occurring repeatedly in the visual pathways anterior to the chiasm, that is in the retina or in the optic nerve or in both. Other conditions, such as hyphaema, intermittent angle closure, ischaemic optic neuropathy (especially in giant cell arteritis), optic disc drusen and papilloedema with obscurations, can cause transient monocular visual loss, but these conditions usually have different durations and clinical different features. Physical effort is a possible trigger of transient monocular visual loss in ocular disorders such as pigmentary glaucoma, Uhthoff phenomenon, reduced retinal arterial flow secondary to vasospasm or internal carotid artery dissection. Other conditions such as retinal venous congestion, hypercoagulable and hyperviscosity conditions, carotid or ophthalmic artery stenosis, chronic ocular hypoperfusion or reduced cardiac output can cause transient monocular visual loss. ${ }^{2-5}$ Repeated transient monocular visual loss during sexual activity has previously been reported only rarely, in relation to subacute angle closure ${ }^{6}$ and to hypothetical retinal vasospasm. ${ }^{7}$ In the latter case, the patient experienced visual loss only during sexual intercourse, not during other physical efforts, and the symptoms disappeared after treatment with nifedipine.

\section{How would you treat the patient?}

The patient was started on oral enalapril $10 \mathrm{mg}$ per day, after which the visual symptoms gradually disappeared over 2 weeks, while resting arterial blood pressure decreased to $120 / 70 \mathrm{~mm} \mathrm{Hg}$.

\section{DISCUSSION}

The physiological response to sexual activity includes an increase in sympathetic nervous system activity, heart rate and systolic blood pressure. ${ }^{8}$ Our patient experienced transient monocular visual loss every time he reached the climax of sexual intercourse, but never while performing strenuous physical exercise. Hypothetical mechanisms of transient monocular visual loss in our patient include vasoconstriction or embolism in the arterial blood supply of the eye. The repeated and completely transient nature of our patient's symptoms supports the fact that embolism was not involved, whereas the resolution of the symptoms after initiation of the treatment with the vasodilator enalapril supports vasoconstriction as the cause of transient blindness. We cannot exclude the possibility that the prior initiation of antithrombotics and statin treatment could have induced an increase in retinal blood flow. ${ }^{9}$

In the present case, treatment with enalapril was chosen based upon the findings of cardiovascular risk factors (atherosclerosis, smoking, hypertension, hyperlipidaemia gender, age). Theoretically, a calcium-channel antagonist, such as nifedipine, would be another attractive treatment option in a condition believed to be precipitated by vasospasms. ${ }^{71}$

In conclusion, transient monocular blindness during sexual intercourse is a symptom that may disclose severe carotid artery disease, its prompt recognition being critical for appropriate therapeutic intervention.

\section{Br J Ophthalmol 2009;93:1250. doi:10.1136/bjo.2007.135780a}

\section{REFERENCES}

1. Barnett HJ, Taylor DW, Eliasziw M, et al. Benefit of carotid endarterectomy in patients with symptomatic moderate or severe stenosis. North American Symptomatic Carotid Endarterectomy Trial Collaborators. New Engl J Med 1998;339:1415-25.

2. Biousse V, Trobe JD. Transient monocular visual loss. Am J Ophthalmol 2005; 140:717-21

3. Ostergaard JR, Kraft M. Benign coital headache. Cephalalgia 1992;12:353-5

4. Schenker HI, Luntz MH, Kels B, et al. Exercise-induced increase of intraocular pressure in the pigmentary dispersion syndrome. Am J Ophthalmol 1980;89:598600.

5. Evans RW, Moore KL. Expert opinion: sexual intercourse followed by headache and transient monocular visual loss. Headache 2008;48:616-20.

6. Patel A, McKibbin M. Exercise-related visual loss in patients with glaucoma. Eye 2003;17:112-13.

7. Teman AJ, Winterkorn JM, Weiner D. Transient monocular blindness associated with sexual intercourse. New Engl J Med 1995:333:393.

8. Giuliano F, Clement P. Neuroanatomy and physiology of ejaculation. Annu Rev Sex Res 2005;16:190-216.

9. Kaiser HJ, Stumpfig D. Flammer J. Short-term effect of dipyridamole on blood flow velocities in the extraocular vessels. Int Ophthalmol 1995;19:355-8.

10. Nagaoka T, Takahashi A, Sato E, et al. Effect of systemic administration of simvastatin on retinal circulation. Arch Ophthalmol 2006:124:665-70.

11. Jehn A, Frank DB, Fleischhauer J, et al. Exercise-induced vasospastic amaurosis fugax. Arch Ophthalmol 2002;120:220-2. 